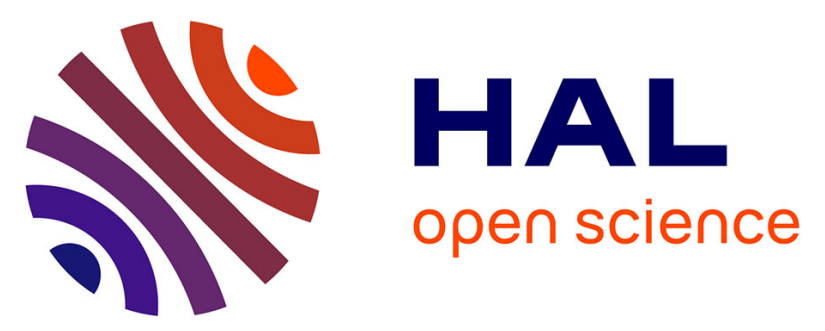

\title{
Strictly Proper Control Design for the Stabilization of 2x2 Linear Hyperbolic ODE-PDE-ODE Systems
}

\author{
David Bou Saba, Federico Bribiesca Argomedo, Michaël Di Loreto, Damien
} Eberard

\section{- To cite this version:}

David Bou Saba, Federico Bribiesca Argomedo, Michaël Di Loreto, Damien Eberard. Strictly Proper Control Design for the Stabilization of 2x2 Linear Hyperbolic ODE-PDE-ODE Systems. 2019 IEEE 58th Conference on Decision and Control (CDC), Dec 2019, Nice, France. pp.4996-5001, 10.1109/CDC40024.2019.9030248 . hal-03498072

\section{HAL Id: hal-03498072 \\ https://hal.science/hal-03498072}

Submitted on 20 Dec 2021

HAL is a multi-disciplinary open access archive for the deposit and dissemination of scientific research documents, whether they are published or not. The documents may come from teaching and research institutions in France or abroad, or from public or private research centers.
L'archive ouverte pluridisciplinaire HAL, est destinée au dépôt et à la diffusion de documents scientifiques de niveau recherche, publiés ou non, émanant des établissements d'enseignement et de recherche français ou étrangers, des laboratoires publics ou privés. 


\title{
Strictly Proper Control Design for the Stabilization of $2 \times 2$ Linear Hyperbolic ODE-PDE-ODE Systems
}

\author{
David Bou Saba ${ }^{1}$, Federico Bribiesca-Argomedo ${ }^{1}$, Michael Di Loreto ${ }^{1}$ and Damien Eberard ${ }^{1}$
}

\begin{abstract}
In this paper, we consider the problem of $L^{2}$ exponential stabilization of a coupled ODE-hyperbolic PDEODE system, where actuation is available through one ODE. Based on the backstepping technique, the system is mapped into an equivalent delay form which allows for the construction of a strictly proper controller realized as a full-state feedback. The result extends previous control designs, lifting some restrictions on the structure of the ODEs under consideration and guaranteeing a non-zero delay margin for the closed-loop system.
\end{abstract}

\section{INTRODUCTION}

Since its introduction [1], the infinite-dimensional backstepping method has been successfully exploited to provide constructive control designs for a wide variety of systems modeled by partial differential equations (PDEs). A good introduction to this method can be found in the textbook [2].

In this paper we are interested in systems modeled by hyperbolic PDEs, an active research topic, an overview of which can be found in [3]. More precisely, we are interested in systems where couplings between the PDEs and some ODE dynamics are present. Many systems of practical interest can be modeled by hyperbolic PDEs. Some examples include drilling applications [4], incompressible flows [5]. Notably, in [6], a backstepping-based output feedback design was experimentally validated in a pneumatic system.

Some previous works considering coupled hyperbolic PDEs and ODEs include [7], for disturbance rejection with a cascaded structure, as well as the observation problem in [8]. For fully interconnected systems (not cascades) some results include [9] for PDE-ODE interconnections, as well as [10], [11] and [12] for ODE-PDE-ODE interconnections and a related result in [13] for a wave equation with dynamic boundary conditions. Recently, however, a great deal of attention has been given to addressing robustness issues present in these designs. Namely, all of these designs, even though mathematically correct, possess a zero robustness margin, as shown in [14]. Delay-robust designs have since been developed for the PDE-ODE interconnection [15].

In this paper, we develop a dynamic full-state feedback control for the stabilization of a class of $2 \times 2$ linear hyperbolic ODE-PDE-ODE systems. Unlike previous control designs for this class of systems, the proposed control law is strictly proper, which guarantees a non-zero delay margin

\footnotetext{
${ }^{1}$ Université de Lyon, INSA Lyon, Laboratoire Ampère (CNRS UMR5005) - F-69621 Villeurbanne, France. \{david.bou-saba, federico.bribiesca, michael.di-loreto, damien.eberard\}einsa-lyon. fr
}

in closed-loop. Furthermore, the assumptions made on the structure of the ODE components are less restrictive than those existing in the literature. In particular, stabilizability instead of controllability is required and the ODE on which the control acts does not need to be of relative degree 1 nor be written in a specific canonical form. Also, since the controller is strictly proper, we can consider weak $L^{2}$ solutions to the PDEs without compatibility conditions on the initial states while still having a continuous control signal.

The paper is organized as follows: In Section II, we state the specific control problem under consideration, as well as the hypotheses required for our control design. In Section III, we present a backstepping transform that allows us to equivalently reformulate the stabilization problem in terms of a target system which, in Section IV, is rewritten in timedelay form. In Section V, a general stabilization result is given for the system as a consequence of the stabilization of a precise output and a particular, constructive, stabilizing controller design for this output is constructed. The resulting controller for the original system can be realized as a strictly proper, dynamic full-state feedback. Finally, Section VI presents numerical simulation results including a small delay in the control input in order to illustrate the proposed control design.

\section{Notation}

Throughout this paper we will use the following notation for a triangular domain in $\mathbb{R}^{2}$

$$
\mathcal{T} \doteq\{(x, y) \in[0,1] \times[0,1], y \geq x\} .
$$

Furthermore, given a set $\Omega \subseteq \mathbb{R}^{2}$, its characteristic function will be denoted

$$
\mathbb{1}_{\{\Omega\}}(x, y) \doteq \begin{cases}1 & \text { if }(x, y) \in \Omega \\ 0 & \text { otherwise. }\end{cases}
$$

The euclidean norm of a vector $\varphi \in \mathbb{R}^{r}, r \in \mathbb{N} \backslash\{0\}$ will be denoted by $\|\varphi\|_{\mathbb{R}^{r}} \doteq\left(\varphi^{\top} \varphi\right)^{1 / 2}$. For a function in $L^{2}([0,1] ; \mathbb{R})$, its norm will be taken in the usual sense

$$
\|\phi\|_{L^{2}}=\left(\int_{0}^{1} \phi^{2}(s) \mathrm{d} s\right)^{1 / 2} .
$$

The notation $I_{r}$ will represent the $r \times r$ identity matrix (if the dimensions are not ambiguous the subindex will be omitted). We denote by $\hat{f}(s)$ the Laplace transform of $f(t)$. For any proper and stable transfer matrix $G(s), \bar{\sigma}(G(j \omega))$ stands for the largest singular value of $G(j \omega)$ at frequency $\omega$, and the $H_{\infty}$-norm of $G$ is

$$
\|G\|_{\infty}=\operatorname{ess} \sup _{\omega \in \mathbb{R}} \bar{\sigma}(G(j \omega)) .
$$




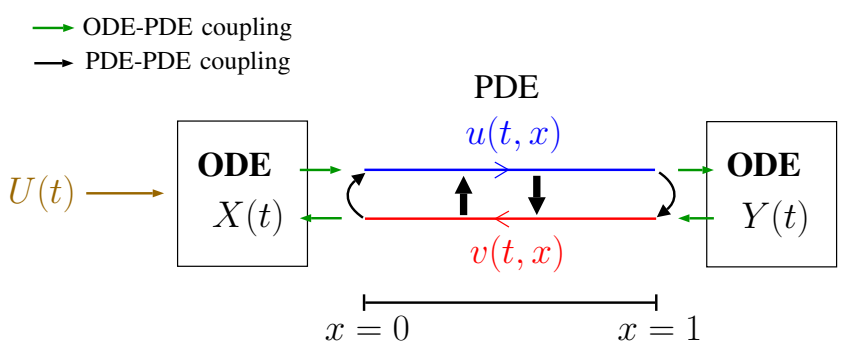

Fig. 1: Schematic representation of the coupled ODE-PDE-ODE system (1)

\section{Problem Statement}

In this paper, we tackle the stabilization problem of a linear interconnected ODE-PDE-ODE system where actuation is only available in one of the component ODEs and the PDE is a $2 \times 2$ hetero-directional hyperbolic PDE, as depicted in Figure 1. This system structure can represent, for example, systems with wave-like propagation between an actuator (with non-negligible dynamics) and a load to be stabilized.

More precisely, we consider systems of the form:

$$
\begin{aligned}
\dot{X}(t) & =A_{0} X(t)+E_{0} v(t, 0)+B_{0} U(t) \\
u(t, 0) & =q v(t, 0)+C_{0} X(t) \\
u_{t}(t, x) & =-\lambda u_{x}(t, x)+\sigma^{+}(x) v(t, x) \\
v_{t}(t, x) & =\mu v_{x}(t, x)+\sigma^{-}(x) u(t, x) \\
v(t, 1) & =\rho u(t, 1)+C_{1} Y(t) \\
\dot{Y}(t) & =A_{1} Y(t)+E_{1} u(t, 1)
\end{aligned}
$$

for a.e. $(t, x) \in[0,+\infty) \times[0,1]$. The states of the system are $(X(t), u(t, \cdot), v(t, \cdot), Y(t)) \in \mathcal{X} \doteq \mathbb{R}^{n} \times$ $L^{2}([0,1] ; \mathbb{R})^{2} \times \mathbb{R}^{m}$ with associated norm $\|(X, u, v, Y)\|_{\mathcal{X}} \doteq$ $\|X\|_{\mathbb{R}^{n}}+\|u\|_{L^{2}}+\|v\|_{L^{2}}+\|Y\|_{\mathbb{R}^{m}}$ (i.e., the norm of each component is the usual Euclidean or $L^{2}$ norm). The control input is $U(t) \in \mathbb{R}^{p}$. The initial conditions are taken as $\left(X_{0}, u_{0}, v_{0}, Y_{0}\right) \in \mathcal{X}$ and we consider weak solutions to (1). In this paper we are interested in exponential stabilization of the system (to zero) in this norm.

The coefficients $\lambda, \mu>0$ represent the transport speeds associated to the two transport equations (convecting in opposite directions). $\sigma^{+}, \sigma^{-}$are sufficiently regular (in this paper, we will assume $\mathcal{C}([0,1] ; \mathbb{R}))$ in-domain couplings between the PDEs. $A_{0} \in \mathbb{R}^{n \times n}, E_{0} \in \mathbb{R}^{n \times 1}, B_{0} \in \mathbb{R}^{n \times p}$, $C_{0} \in \mathbb{R}^{1 \times n}, q \in \mathbb{R}, A_{1} \in \mathbb{R}^{m \times m}, E_{1} \in \mathbb{R}^{m \times 1}, C_{1} \in \mathbb{R}^{1 \times m}$, and $\rho \in \mathbb{R}$.

Note that the results in this paper could be extended to space-dependent transport speeds in a similar manner to [16]. For simplicity, we have chosen to consider the constant transport speed case. The fact that we do not consider diagonal coupling terms in the PDEs is not restrictive, since, via a change of variables like that in [17], these coefficients can be transferred to the anti-diagonal terms.

In order to design our controller, the following assumptions are made:

Assumption 1: The coefficients $\rho, q$ satisfy $|\rho q|<1$ and $\rho \neq 0$.
Assumption 2: The pairs $\left(A_{0}, B_{0}\right)$ and $\left(A_{1}, E_{1}\right)$ are stabilizable (i.e. there exist $F_{0} \in \mathbb{R}^{p \times n} F_{1} \in \mathbb{R}^{1 \times m}$ such that $\bar{A}_{0} \doteq A_{0}+B_{0} F_{0}$ and $\bar{A}_{1} \doteq A_{1}+E_{1} F_{1}$ are Hurwitz).

Assumption 3: The matrices $\left(A_{0}, B_{0}, C_{0}\right)$ satisfy

$$
\operatorname{rank}\left(\left[\begin{array}{cc}
s I-A_{0} & B_{0} \\
C_{0} & 0_{1 \times p}
\end{array}\right]\right)=n+1
$$

for all $s \in \mathbb{C}, \operatorname{Re}(s) \geq 0$.

Remark that the first condition in Assumption $1(|\rho q|<1)$ is required in order to be able to robustly stabilize system (1) in the sense of [18], [14], [15]. If this condition is not verified, any linear control law developed for the system will lead to a zero delay margin. The second condition in this Assumption $(\rho \neq 0)$ is required by the structure of the backstepping transform and target systems chosen in this paper and does not seem particularly restrictive in an application since, for a wave-like equation, a zero reflection requires perfect impedance matching between the PDE and the ODE load at the end. It is possible that the method described in this paper can be adapted for this case, but it is outside the scope of the current article.

Assumption 2 is not overly conservative since without the stabilizability of $\left(A_{1}, E_{1}\right)$, it becomes impossible to ask for stabilization of the $Y$ subsystem in (1) independently of the PDE or interconnection structure. The stabilizability assumption on $\left(A_{0}, B_{0}\right)$ allows for a simpler stabilizing design (no modes of the $X$ subsystem are stabilized indirectly through the load) and to set conditions that can be easily tested.

Assumption 3 serves several purposes. In particular it implies that $C_{0}$ is not identically zero (which would obstruct the stabilization of the PDE and $Y$ subsystems through $X$ ). It is also equivalent (under Assumption 2) to asking that the transfer matrix $P_{0}(s) \doteq C_{0}\left(s I-\bar{A}_{0}\right)^{-1} B_{0}$ (with $\bar{A}_{0}$ defined in Assumption 2) does not have any zeros in the complex right-half plane that are common to all its components. This Assumption is directly used in the constructive design of a control law and can be tested in a simple way (either in the matrix form or in the equivalent transfer matrix form $P_{0}$ ). More details on the relevance and conservatism of this hypothesis will be given in Section V. It is less restrictive than other hypotheses in the literature in terms of relative degree of the actuator (such as invertibility of $B_{0}$ [10] or of $C_{0} B_{0}[11]$ ), extends naturally to multi-input systems and does not require the system to be written in any particular form [12].

\section{Backstepping Transform And Delay System}

\section{A. Backstepping Transform}

In order to stabilize system (1) we begin by transforming it using the backstepping method. We need to find a linear bounded (and boundedly invertible) transform

$$
\begin{aligned}
X(t)= & \xi(t)+\int_{0}^{1} K^{12}(y) \alpha(t, y) \mathrm{d} y \\
& +\int_{0}^{1} K^{13}(y) \beta(t, y) \mathrm{d} y+K^{14} \eta(t)
\end{aligned}
$$




$$
\begin{aligned}
u(t, x)= & \alpha(t, x)+\int_{x}^{1} K^{22}(x, y) \alpha(t, y) \mathrm{d} y \\
& +\int_{x}^{1} K^{23}(x, y) \beta(t, y) \mathrm{d} y+K^{24}(x) \eta(t) \\
v(t, x)= & \beta(t, x)+\int_{x}^{1} K^{32}(x, y) \alpha(t, y) \mathrm{d} y \\
& +\int_{x}^{1} K^{33}(x, y) \beta(t, y) \mathrm{d} y+K^{34}(x) \eta(t) \\
Y(t)= & \eta(t)
\end{aligned}
$$

that maps a target system

$$
\begin{aligned}
\dot{\xi}(t)= & \bar{A}_{0} \xi(t)-\lambda K^{12}(0) C_{0} \xi(t)+\bar{E}_{1} \alpha(t, 1) \\
& +\bar{E}_{0} \beta(t, 0)+M \eta(t)+B_{0} \tilde{U}(t) \\
& +\int_{0}^{1} M^{\alpha}(y) \alpha(t, y) \mathrm{d} y+\int_{0}^{1} M^{\beta}(y) \beta(t, y) \mathrm{d} y \\
\alpha(t, 0)= & q \beta(t, 0)+C_{0} \xi(t) \\
\alpha_{t}(t, x)= & -\lambda \alpha_{x}(t, x) \\
\beta_{t}(t, x)= & \mu \beta_{x}(t, x) \\
\beta(t, 1)= & \rho \alpha(t, 1) \\
\dot{\eta}(t)= & \bar{A}_{1} \eta(t)+E_{1} \alpha(t, 1)
\end{aligned}
$$

with corresponding initial conditions $\left(\xi_{0}, \alpha_{0}, \beta_{0}, \eta_{0}\right) \in \mathcal{X}$ into (1). $\bar{A}_{0}$ and $\bar{A}_{1}$ are taken as defined in Assumption 2 and the other coefficients are defined as

$$
\begin{aligned}
\bar{E}_{0} & =E_{0}-q \lambda K^{12}(0)+\mu K^{13}(0) \\
\bar{E}_{1} & =\lambda K^{12}(1)-K^{14} E_{1}-\rho \mu K^{13}(1) \\
M & =A_{0} K^{14}-K^{14} \bar{A}_{1}+E_{0} K^{34}(0) \\
M^{\alpha}(y) & =E_{0} K^{32}(0, y)+A_{0} K^{12}(y)-\lambda K_{y}^{12}(y) \\
M^{\beta}(y) & =E_{0} K^{33}(0, y)+A_{0} K^{13}(y)+\mu K_{y}^{13}(y) .
\end{aligned}
$$

The new control input $\tilde{U}$ in (3a) is defined as

$$
\tilde{U}(t) \doteq U(t)-F_{0} \xi(t) .
$$

Following the backstepping procedure, we find that the backstepping kernels must satisfy the following PDEs

$$
\begin{aligned}
& \lambda K_{x}^{22}(x, y)+\lambda K_{y}^{22}(x, y)=\sigma^{+}(x) K^{32}(x, y) \\
& \lambda K_{x}^{23}(x, y)-\mu K_{y}^{23}(x, y)=\sigma^{+}(x) K^{33}(x, y) \\
& \mu K_{x}^{32}(x, y)-\lambda K_{y}^{32}(x, y)=-\sigma^{-}(x) K^{22}(x, y) \\
& \mu K_{x}^{33}(x, y)+\mu K_{y}^{33}(x, y)=-\sigma^{-}(x) K^{23}(x, y)
\end{aligned}
$$

with boundary conditions

$$
\begin{aligned}
& K^{22}(x, 1)=\frac{1}{\lambda}\left(K^{24}(x) E_{1}+\rho \mu K^{23}(x, 1)\right) \\
& K^{23}(x, x)=-\frac{\sigma^{+}(x)}{\lambda+\mu} \\
& K^{32}(x, x)=\frac{\sigma^{-}(x)}{\lambda+\mu} \\
& K^{33}(x, 1)=\frac{1}{\mu \rho}\left(\lambda K^{32}(x, 1)-K^{34}(x) E_{1}\right)
\end{aligned}
$$

as well as the set of ODEs

$$
\begin{aligned}
& K_{x}^{24}(x)=\frac{1}{\lambda}\left(-K^{24}(x) \bar{A}_{1}+\sigma^{+}(x) K^{34}(x)\right) \\
& K_{x}^{34}(x)=\frac{1}{\mu}\left(K^{34}(x) \bar{A}_{1}-\sigma^{-}(x) K^{24}(x)\right)
\end{aligned}
$$

with boundary conditions

$$
\begin{aligned}
& K^{24}(1)=F_{1} \\
& K^{34}(1)=C_{1}+\rho F_{1} .
\end{aligned}
$$

And, finally, the set of algebraic relations are fulfilled

$$
\begin{aligned}
C_{0} K^{12}(y) & =K^{22}(0, y)-q K^{32}(0, y) \\
C_{0} K^{13}(y) & =K^{23}(0, y)-q K^{33}(0, y) \\
C_{0} K^{14} & =K^{24}(0)-q K^{34}(0) .
\end{aligned}
$$

Notice in particular that a solution to the set of algebraic equations can be obtained using the Moore-Penrose rightinverse of $C_{0}, C_{0}^{+} \doteq C_{0}^{T}\left(C_{0} C_{0}^{T}\right)^{-1}$. This can be done since, by Assumption 3, $C_{0}$ is necessarily full-row rank (with rank equal to 1). In the remainder of the article, we solve these algebraic relations using this right-inverse (which makes the solution unique).

This set of PDEs and ODEs have a unique continuous solution in their respective domains: $K^{22}, K^{23}, K^{32}, K^{33} \in$ $\mathcal{C}(\mathcal{T} ; \mathbb{R})$ and $K^{24}, K^{34} \in \mathcal{C}\left([0,1] ; \mathbb{R}^{1 \times p}\right)$ (row vectors). This follows, with minor adaptations, from the results in [9] and [19] and the regularity of the coefficients (the required adaptations in the proof are similar in spirit to those in [10], even though the kernel equations obtained here are simpler). More regularity can be obtained, if necessary, by increasing the regularity of the coefficients. For the purposes of this article, continuous solutions are enough for all the terms in the transform and the target system to be adequately defined. Then, we can solve equation (10) using the Moore-Penrose right-inverse to obtain a specific value for $K^{12}, K^{13} \in$ $\mathcal{C}\left([0,1] ; \mathbb{R}^{n \times 1}\right)$ (column vectors) and $K^{14} \in \mathbb{R}^{n \times m}$.

\section{B. Boundedness and Invertibility of the Transform}

Boundedness of the transform is a direct consequence of the structure of the transform (identities, integral operators and matrices) and the regularity of the backstepping kernels (continuous in this case). As for invertibility, it follows from the structure of the transform, which is block uppertriangular with the blocks on the diagonal being either identities (for the ODEs) or (invertible) Volterra operators (for the PDEs). The inverse transform will again present a block upper-triangular structure analogous to the one of the direct transform. The computation of the inverse transform follows standard backstepping procedures and will not be detailed in this article.

\section{TARGET SYSTEM IN DELAY FORM}

Using the method of characteristics, the solution of (3c) and (3d) satisfies, for any $t>\max \left(\frac{1}{\lambda}, \frac{1}{\mu}\right)$ and $x \in[0,1]$, 


$$
\begin{aligned}
& \alpha(t, x)=\alpha\left(t-\frac{x}{\lambda}, 0\right) \\
& \beta(t, x)=\beta\left(t-\frac{1-x}{\mu}, 1\right) .
\end{aligned}
$$

Substituting these expressions in (3b) and (3e), and denoting $\tau=\frac{1}{\mu}+\frac{1}{\lambda}$, we obtain, for any $t>\tau$,

$$
\begin{aligned}
& \alpha(t, 0)=\rho q \alpha(t-\tau, 0)+C_{0} \xi(t), \\
& \beta(t, 1)=\rho q \beta(t-\tau, 1)+\rho C_{0} \xi\left(t-\frac{1}{\lambda}\right) .
\end{aligned}
$$

In the target system, the transport equations are then equivalent to a set of two continuous-time difference equations acting on the boundaries and coupled to the state $\xi(t)$. The stabilizing control will be designed in the frequency domain, using Laplace transform. For brevity, and without any loss of generality for the asymptotic stable behavior of the plant, we assume all zero initial conditions. The Laplace transform applied to (12) leads to

$$
\begin{aligned}
& \left(1-\rho q \mathrm{e}^{-\tau s}\right) \hat{\alpha}(s, 0)=C_{0} \hat{\xi}(s) \\
& \left(1-\rho q \mathrm{e}^{-\tau s}\right) \hat{\beta}(s, 1)=\rho C_{0} \mathrm{e}^{-\frac{s}{\lambda}} \hat{\xi}(s) .
\end{aligned}
$$

The Laplace transform of (3f) writes as

$$
\left(s I-\bar{A}_{1}\right) \hat{\eta}(s)=\mathrm{e}^{-\frac{s}{\lambda}} E_{1} \hat{\alpha}(s, 0) .
$$

The Laplace transform of (3a) becomes, incorporating therein (11), (13) and (14),

$$
\begin{aligned}
(1- & \left.\rho q \mathrm{e}^{-\tau s}\right)\left(s I-\bar{A}_{0}\right) \hat{\xi}(s) \\
= & -\left(1-\rho q \mathrm{e}^{-\tau s}\right) \lambda K^{12}(0) C_{0} \hat{\xi}(s)+\mathrm{e}^{-\frac{s}{\lambda}} \bar{E}_{1} C_{0} \hat{\xi}(s) \\
& +\rho \mathrm{e}^{-\tau s} \bar{E}_{0} C_{0} \hat{\xi}(s)+M\left(s I-\bar{A}_{1}\right)^{-1} E_{1} \mathrm{e}^{-\frac{s}{\lambda}} C_{0} \hat{\xi}(s) \\
& +\int_{0}^{\frac{1}{\lambda}} \lambda M^{\alpha}(\lambda \theta) \mathrm{e}^{-s \theta} \mathrm{d} \theta C_{0} \hat{\xi}(s) \\
& +\int_{0}^{\frac{1}{\mu}} \rho \mu M^{\beta}(1-\mu \theta) \mathrm{e}^{-s \theta} \mathrm{d} \theta \mathrm{e}^{-\frac{s}{\lambda}} C_{0} \hat{\xi}(s) \\
& +\left(1-\rho q \mathrm{e}^{-\tau s}\right) B_{0} \hat{\tilde{U}}(s) .
\end{aligned}
$$

From Assumption 1, the quasipolynomial $\left(1-\rho q \mathrm{e}^{-\tau s}\right)$ is nonsingular for any $s \in \mathbb{C}, \operatorname{Re}(s) \geq 0$, as well as is the matrix polynomial $\left(s I-\bar{A}_{1}\right)$ from Assumption 2. Using these invertibility properties, identity (15) can be written equivalently, for any $s \in \mathbb{C}$ with $\operatorname{Re}(s) \geq 0$, as

$$
\left(s I-\bar{A}_{0}\right) \hat{\xi}(s)=G(s) C_{0} \hat{\xi}(s)+B_{0} \hat{\tilde{U}}(s),
$$

where

$$
\begin{aligned}
& G(s)=-\lambda K^{12}(0) \\
&+\left(1-\rho q \mathrm{e}^{-\tau s}\right)^{-1} {\left[\left(\bar{E}_{1}+M\left(s I-\bar{A}_{1}\right)^{-1} E_{1}\right) \mathrm{e}^{-\frac{s}{\lambda}}\right.} \\
&\left.+\rho \mathrm{e}^{-\tau s} \bar{E}_{0}+\int_{0}^{\tau} M^{\xi}(\theta) \mathrm{e}^{-s \theta} \mathrm{d} \theta\right]
\end{aligned}
$$

and

$$
\begin{aligned}
M^{\xi}(\theta)= & \lambda M^{\alpha}(\lambda \theta) \mathbb{1}_{\left[0, \frac{1}{\lambda}\right]}(\theta) \\
& +\rho \mu M^{\beta}\left(1-\mu \theta+\frac{\mu}{\lambda}\right) \mathbb{1}_{\left(\frac{1}{\lambda}, \tau\right]}(\theta) .
\end{aligned}
$$

\section{TARGET SYSTEM STABILIZATION}

The objective of this section is the design of a stabilizing control law for the target system, described by (16). As a preliminary remark, a well-known necessary and sufficient condition for (spectral) stabilization of the target plant is that

$$
\operatorname{rank}\left[s I-\bar{A}_{0}-G(s) C_{0} \quad B_{0}\right]=n,
$$

for all $s \in \mathbb{C}, \operatorname{Re}(s) \geq 0$ [20]. The transfer function matrix $G(s)$ in (17) is related to the interconnection of a neutral time-delay system (with stable difference operator) and an LTI system, where the interconnection involves pointwise and distributed delays, the last having a kernel which is realized numerically solving a set of PDEs. This complexity makes this stabilization condition not reliable for numerical analysis and constructive design purposes. In order to overcome the intrinsic difficulties related to spectral stabilization, we propose to decompose the problem in two steps. First, we deal with the stabilization problem for the output of the first ODE subsystem, namely $y(t) \doteq C_{0} \xi(t)$. Then, we show that exponential stabilization of this output implies exponential stabilization of the whole target system (which, based on the properties of the backstepping transform is equivalent to exponential stabilization of the original system).

Since we defined $y(t) \doteq C_{0} \xi(t)$, we have that, using (16),

$$
\hat{y}(s)=C_{0}\left(s I-\bar{A}_{0}\right)^{-1} G(s) \hat{y}(s)+P_{0}(s) \hat{\tilde{U}}(s)
$$

where

$$
P_{0}(s) \doteq C_{0}\left(s I-\bar{A}_{0}\right)^{-1} B_{0} .
$$

The rank of the matrix in Assumption 3 is conserved for the triple $\left(\bar{A}_{0}, B_{0}, C_{0}\right)$ since

$$
\left[\begin{array}{cc}
s I-\bar{A}_{0} & B_{0} \\
C_{0} & 0
\end{array}\right]=\left[\begin{array}{cc}
s I-A_{0} & B_{0} \\
C_{0} & 0
\end{array}\right]\left[\begin{array}{cc}
I & 0 \\
-F_{0} & I
\end{array}\right] .
$$

Furthermore, using the following expression

$$
\left[\begin{array}{cc}
s I-\bar{A}_{0} & B_{0} \\
C_{0} & 0
\end{array}\right]=\left[\begin{array}{cc}
I & G(s) \\
0 & I
\end{array}\right]\left[\begin{array}{cc}
s I-\bar{A}_{0}-G(s) C_{0} & B_{0} \\
C_{0} & 0
\end{array}\right],
$$

we can conclude that Assumption 3 implies that the aforementioned necessary and sufficient condition holds, i.e.

$$
\operatorname{rank}\left[s I-\bar{A}_{0}-G(s) C_{0} \quad B_{0}\right]=n,
$$

for all $s \in \mathbb{C}, \operatorname{Re}(s) \geq 0$.

Notice that Assumption 3 is equivalent to the existence of a right inverse for $P_{0}(s)$ defined in (20), whose entries have no unstable poles (remark that such an inverse is not proper) [21]. We denote $P_{0}^{+}(s)$ any such right inverse. A possible choice is given by the Moore-Penrose right inverse $P_{0}^{+}(s)=P_{0}^{T}(s)\left(P_{0}(s) P_{0}^{T}(s)\right)^{-1}$.

Before proceeding with the construction of a stabilizing feedback for the output $y(t)$, we state the following stability result for the target system.

Theorem 1: Assume that $y(\cdot)$ is exponentially stabilized by a dynamic output feedback of the form

$$
\hat{\tilde{U}}(s)=F(s) \hat{y}(s),
$$


where $F(s)$ is a stable (proper) transfer matrix. Then, under Assumptions 1-3, the target system (3) is exponentially stable in the $\mathcal{X}$-norm.

Proof: Assume that $y(\cdot)$ is exponentially stable. From (16), the closed-loop dynamics for $\xi(\cdot)$ are described by

$$
\hat{\xi}(s)=\left(s I-\bar{A}_{0}\right)^{-1}\left(G(s)+B_{0} F(s)\right) \hat{y}(s) .
$$

By Assumption 2, $\bar{A}_{1}$ is Hurwitz and, by Assumption 1, $\left(1-\rho q \mathrm{e}^{-\tau s}\right)$ has a stable inverse. It follows that $G(s)$ in (17) is a stable, proper transfer matrix, as is $F(s)$. Since Assumption 2 also guarantees that $\bar{A}_{0}$ is Hurwitz, stabilization of $\xi(\cdot)$ is implied by the previous equality. The stability of $\alpha(\cdot, 0), \beta(\cdot, 1)$ is deduced from (13), which implies exponential convergence to zero of $\alpha$ and $\beta$ in the $L^{2}$-norm (this can be seen using (11)). Stability of $\eta(\cdot)$ is obtained from identity (14).

In the following, we describe the construction of a stabilizing output feedback of the form (21). For this, we decompose $G(s)$ in (17) into

$$
G(s)=\mathrm{w}(s) G(s)+(1-\mathrm{w}(s)) G(s),
$$

with $\mathrm{w}(s)$ a (SISO) stable low-pass filter of sufficient order to be designed. A candidate (proper) controller can therefore be defined as

$$
F(s)=-P_{0}^{+}(s)\left[C_{0}\left(s I-\bar{A}_{0}\right)^{-1} \mathrm{w}(s) G(s)\right] .
$$

We show in the following result that the low-pass filter $\mathrm{w}(s)$ can always be chosen in order to make $F(s)$ a stabilizing controller for $y(\cdot)$.

Proposition 2: Let $\mathrm{w}(s)$ be any low-pass filter, with sufficiently high relative degree, such that

$$
\forall \omega \in \mathbb{R},|1-\mathrm{w}(j \omega)|<\frac{1}{\|G\|_{\infty} \bar{\sigma}\left(C_{0}\left(j \omega I-\bar{A}_{0}\right)^{-1}\right)} .
$$

Then the dynamic output feedback (21) with $F(s)$ given in (24) exponentially stabilizes $y(\cdot)$.

Proof: First, remark that the relative degree of $\mathrm{w}(s)$ can always be chosen such that $F(s)$ in (24) is strictly proper. Plugging (23)-(24) into (19), the closed-loop dynamics of $y(\cdot)$ is governed by

$$
(1-\Phi(s)) \hat{y}(s)=0
$$

where

$$
\Phi(s) \doteq(1-\mathrm{w}(s)) C_{0}\left(s I-\bar{A}_{0}\right)^{-1} G(s) .
$$

Since $G(s)$ given in (17) is uniformly bounded in the righthalf complex plane, we have $\bar{\sigma}(G(j \omega)) \leq\|G\|_{\infty}$ for all $\omega$. Noting that $\Phi(s)$ is stable and strictly proper $\left(\bar{A}_{0}\right.$ is Hurwitz by Assumption 2), we have by (25) that

$$
\begin{aligned}
\bar{\sigma}(\Phi(j \omega)) & \leq|1-\mathrm{w}(j \omega)|\|G\|_{\infty} \bar{\sigma}\left(C_{0}\left(j \omega I-\bar{A}_{0}\right)^{-1}\right) \\
& <1
\end{aligned}
$$

for all $\omega \in \mathbb{R}$. This implies that $\|\Phi\|_{\infty}<1$, which is a sufficient condition for exponential stability of $y(\cdot)$ in (26).
Theorem 1 and Proposition 2 prove that there exists a dynamic feedback in the form (21) such that the target system (3) is exponentially stable, that is $\|(\xi, \alpha, \beta, \eta)\|_{\mathcal{X}}$ converges exponentially to zero for any initial condition in $\mathcal{X}$.

Given a stabilizing control input $\tilde{U}$ for the target system, the corresponding control input for the original system (1) can be obtained from (5) as

$$
U(t)=\tilde{U}(t)+F_{0} \xi(t) .
$$

The implementation of this controller requires some realization of $F(s)$ in (24), as well as the inverse of the backstepping transform (2) in order to reconstruct $\xi$ and $y$ from the original system variables $(X, u, v, Y) \in \mathcal{X}$.

Remark that the controller has been chosen as strictly proper (we do not use the image of $C_{0} B_{0}$ to compensate any terms directly) which means that our controller is robust to small delays in the input, which is not the case in some designs that include derivative terms. The proof follows the same ideas as that in [14].

\section{NumERiCAL Simulation}

The proposed control was simulated using Matlab and Simulink. The transport PDEs were discretized using an explicit in time, first-order, upwind finite difference method with 101 spatial discretization points (and a CFL number of $0.5)$. The transfer functions in the control law were transformed to a state-space representation for implementation. The numerical values used were: $\lambda=2, \mu=0.7, \sigma^{+}=1$, $\sigma^{-}=0.5, \rho=0.5, q=1.2$,

$$
\begin{aligned}
& A_{0}=\left[\begin{array}{cccc}
0 & 0.14 & 0 & 0.1 \\
0 & 0 & 0.14 & 0 \\
0.29 & -0.43 & 0.57 & 0.2 \\
0 & 0 & 0 & -1.1
\end{array}\right], B_{0}=\left[\begin{array}{cc}
0 & 0 \\
0 & -1 \\
1 & -1 \\
0 & 0
\end{array}\right] \text {, } \\
& C_{0}=\left[\begin{array}{llll}
1 & 0 & 0 & -0.5
\end{array}\right], E_{0}=\left[\begin{array}{c}
2 \\
-1 \\
0.1 \\
0
\end{array}\right] \\
& F_{0}=\left[\begin{array}{cccc}
41.71 & 5.43 & -1.93 & 0 \\
42 & 5 & 0.14 & 0
\end{array}\right] \text {, } \\
& A_{1}=\left[\begin{array}{ccc}
0.29 & 0.14 & 0 \\
0.14 & 0 & 0.1 \\
0 & 0 & -0.9
\end{array}\right], E_{1}=\left[\begin{array}{c}
-1 \\
1 \\
0
\end{array}\right] \\
& C_{1}=\left[\begin{array}{lll}
0 & 1 & 0.5
\end{array}\right], F_{1}=\left[\begin{array}{lll}
12 & 8.71 & 0
\end{array}\right] \text {. }
\end{aligned}
$$

Notice that the system verifies Assumptions 1-3 and that $C_{0} B_{0}=\left[\begin{array}{ll}0 & 0\end{array}\right]$. Furthermore, the ODE systems are only stabilizable in this case and need not be transformed into any particular standard form. Also, each ODE and the PDE subsystem are independently unstable (and remain so when interconnected). Also, an input delay of $0.025 \mathrm{~s}$ was introduced in the control action to show the robustness of the design to small delays in the loop. The chosen $\mathrm{w}(s)$ is a simple 4th order low-pass filter with a bandwidth of approximately $100 \mathrm{rad} / \mathrm{s}$. 


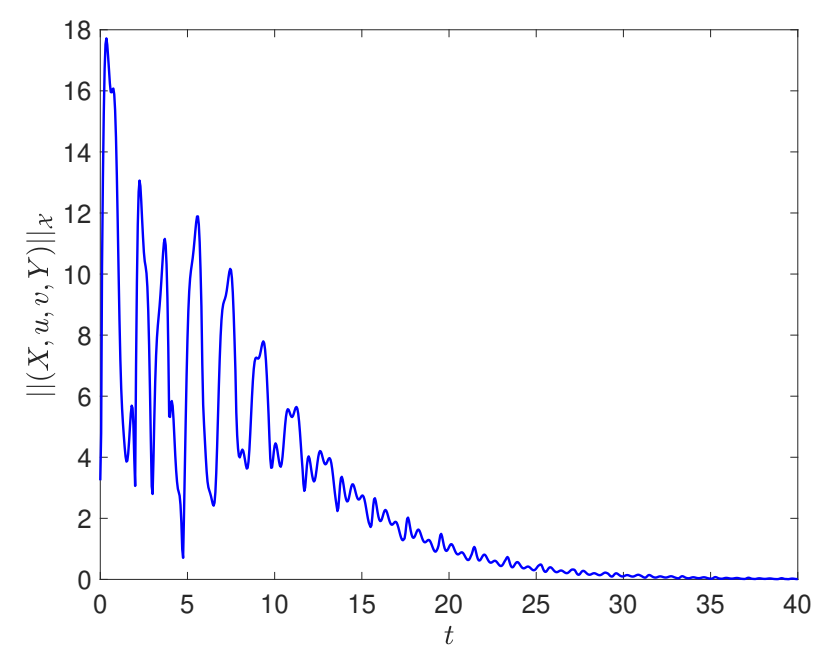

Fig. 2: Evolution of the norm $\|(X, u, v, Y)\|_{\mathcal{X}}$ of the states in closed-loop with an input delay of $0.025 \mathrm{~s}$.

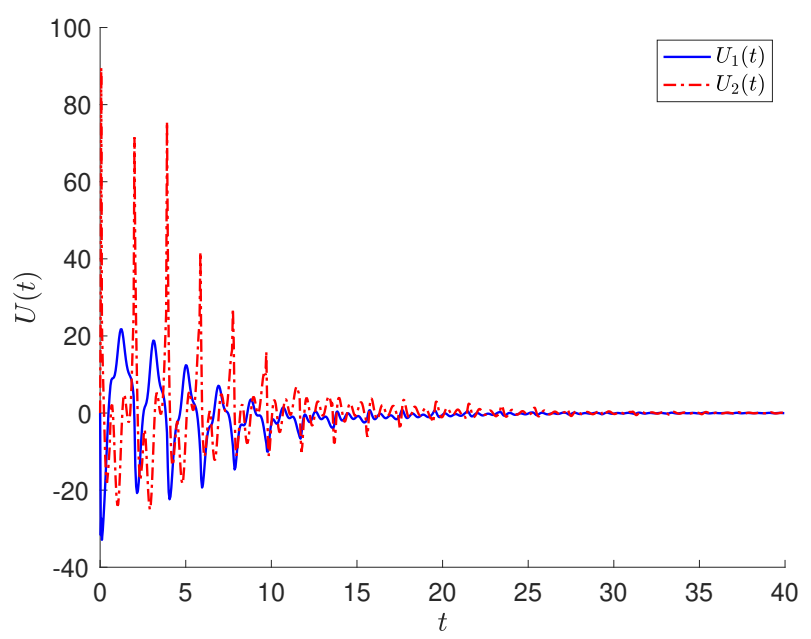

Fig. 3: Evolution of the control input $U$ in closed-loop with an input delay of $0.025 \mathrm{~s}$

The states of the system converge to zero, as expected, despite the input delay. The evolution of the norm $\|(X, u, v, Y)\|_{\mathcal{X}}$ of the states is given in Figure 2 . The control action is shown in Figure 3. It is interesting to notice that, unlike in [10], we no longer require any compatibility in the initial conditions for the ODEs and PDEs in order to guarantee a continuous control signal.

\section{Conclusion And PERspectives}

In this paper, a strictly proper dynamic full-state feedback controller was designed for the stabilization of a class of $2 \times 2$ linear hyperbolic ODE-PDE-ODE systems. The proposed approach is based on a backstepping transform that allows us to reformulate the stabilization problem in terms of a time-delay system with pointwise and distributed delays. By restricting the bandwidth of the control with an adequate low-pass filter we are able to guarantee the delay-robustness of the resulting controller. Future works will focus on the extension of these results to the output-feedback case.

\section{REFERENCES}

[1] A. Balogh and M. Krstic, "Infinite dimensional backstepping-style feedback transformations for a heat equation with an arbitrary level of instability," European journal of control, vol. 8, no. 2, pp. 165-175, 2002.

[2] M. Krstic and A. Smyshlyaev, Boundary control of PDEs: A course on backstepping designs. Siam, 2008, vol. 16.

[3] G. Bastin and J.-M. Coron, Stability and boundary stabilization of 1-D hyperbolic systems, ser. Progress in Nonlinear Differential Equations and Their Applications Subseries in Control. Birkhäuser, 2016, vol. 88 .

[4] C. Sagert, F. Di Meglio, M. Krstic, and P. Rouchon, "Backstepping and flatness approaches for stabilization of the stick-slip phenomenon for drilling," IFAC Proceedings Volumes, vol. 46, no. 2, pp. 779-784, 2013.

[5] F. Castillo, E. Witrant, C. Prieur, and L. Dugard, "Boundary observers for linear and quasi-linear hyperbolic systems with application to flow control," Automatica, vol. 49, no. 11, pp. 3180-3188, 2013.

[6] R. Kern, N. Gehring, J. Deutscher, and J. Meissner, "Design and experimental validation of an output feedback controller for a pneumatic system with distributed parameters," in 18th International Conference on Control, Automation and Systems (ICCAS), South Korea, 2018, pp. 1391-1396.

[7] O. Aamo, "Disturbance rejection in $2 \times 2$ linear hyperbolic systems," IEEE Transactions on Automatic Control, vol. 58, no. 5, pp. 10951106, May 2013.

[8] A. Hasan, O. Aamo, and M. Krstic, "Boundary observer design for hyperbolic PDE-ODE cascade systems," Automatica, vol. 68, pp. 7586, 2016.

[9] F. Di Meglio, F. Bribiesca Argomedo, L. Hu, and M. Krstic, "Stabilization of coupled linear heterodirectional hyperbolic PDE-ODE systems," Automatica, vol. 87, pp. 281-289, 2018.

[10] D. Bou Saba, F. Bribiesca Argomedo, M. Di Loreto, and D. Eberard, "Backstepping stabilization of $2 \times 2$ linear hyperbolic PDEs coupled with potentially unstable actuator and load dynamics," in 56th IEEE Conference on Decision and Control (CDC), December 2017, pp. 2498-2503.

[11] J. Deutscher, N. Gehring, and R. Kern, "Output feedback control of general linear heterodirectional hyperbolic ODE-PDE-ODE systems," Automatica, vol. 95, pp. 472-480, September 2018.

[12] J. Wang, M. Krstic, and Y. Pi, "Control of a $2 \times 2$ coupled linear hyperbolic system sandwiched between 2 ODEs," International Journal of Robust and Nonlinear Control, vol. 28, pp. 3987-4016, 2018.

[13] C. Roman, D. Bresch-Pietri, E. Cerpa, C. Prieur, and O. Sename, "Backstepping control of a wave PDE with unstable source terms and dynamic boundary," IEEE Control Systems Letters, vol. 2, no. 3, pp. 459-464, 2018.

[14] J. Auriol, U. J. F. Aarsnes, P. Martin, and F. Di Meglio, "Delay-robust control design for heterodirectional linear coupled hyperbolic PDEs," IEEE Transactions on Automatic Control, 2018.

[15] J. Auriol, F. Bribiesca Argomedo, D. Bou Saba, M. Di Loreto, and F. Di Meglio, "Delay-robust stabilization of a hyperbolic PDE-ODE system," Automatica, vol. 95, pp. 494-502, 2018.

[16] J.-M. Coron, R. Vazquez, M. Krstic, and G. Bastin, "Local exponential $H^{2}$ stabilization of a $2 \times 2$ quasilinear hyperbolic system using backstepping," SIAM Journal on Control and Optimization, vol. 51, no. 3, pp. 2005-2035, 2013.

[17] G. Bastin and J.-M. Coron, "Further results on boundary feedback stabilisation of $2 \times 2$ hyperbolic systems over a bounded interval," in 8th IFAC Symposium on Nonlinear Control Systems, Bologna, Italy, September 2010, pp. 1081-1085.

[18] J. Hale and S. V. Lunel, "Strong stabilization of neutral functional differential equations," IMA Journal of Mathematical Control and Information, vol. 19, no. 1 and 2, pp. 5-23, 2002.

[19] L. Hu, F. Di Meglio, R. Vazquez, and M. Krstic, "Boundary exponential stabilization of 1-D inhomogeneous quasilinear hyperbolic systems," arXiv preprint arXiv:1512.03539, 2015.

[20] L. Pandolfi, "Stabilization of neutral functional differential equations," J. Optimization Theory and Applications, vol. 20, no. 2, pp. 191-204, 1976.

[21] P. Moylan, "Stable inversion of linear systems," IEEE Transactions on Automatic Control, vol. 22, no. 1, pp. 74-78, 1977. 